\title{
Direct measurements of mRNA translation kinetics in living cells
}

\author{
Mikhail Metelev, Ivan L. Volkov, Erik Lundin, Arvid H. Gynnå, Johan Elf, and \\ Magnus Johansson* \\ Department of Cell and Molecular Biology, Uppsala University \\ *Correspondence: m.johansson@icm.uu.se
}

\section{ABSTRACT}

Ribosome mediated mRNA translation is central to life as we know it. The cycle of translation has, however, not been characterized in a living cell. Here we have developed a live-cell ribosomelabeling method, which allows us to characterize the whole processes of finding an mRNA and translating it, using single-molecule tracking techniques. We find that more than $90 \%$ of both bacterial ribosomal subunits are engaged in elongation at any particular time, and that neither of the subunits, in general, continues translation from one open reading frame to the next on a poly-cistronic mRNA. Furthermore, we find that a variety of previously published orthogonal ribosomes, with altered anti-Shine-Dalgarno sequences, show significant binding to endogenous mRNAs, with the rate of translation initiation only modestly affected. Hence, our results suggest that other mRNA elements than the SD sequence play major roles in directing the ribosome to the correct translation start sites.

\section{INTRODUCTION}

Translation of mRNAs into proteins is one of the most fundamental molecular processes in all living organisms. Central to this process is the ribosome, a highly conserved macromolecular machine consisting of two subunits, the small ( $30 \mathrm{~S}$ in bacteria), and the large (50S in bacteria). mRNA translation has been extensively studied over the decades, and a wide variety of bulk biochemical approaches and structural techniques have helped painting a detailed picture of the 
process $^{1}$. Recent advances in cryogenic electron microscopy continue to provide new snapshots of ribosomes with numerous ligands, capturing multiple conformations, and revealing hidden structural dynamics ${ }^{2-3}$. Emerging single-molecule techniques have, at the same time, helped to connect the conformational dynamics of the ribosome to transient interactions with ligands ${ }^{4}$. Until now, however, most studies of translation kinetics have been performed using reconstituted in vitro systems. Although extremely powerful, studies of an isolated system can never fully account for the complexity emerging from inter-connected molecular processes occurring in the crowded environment of the living cell. The development of new methods that allow direct measurements of mRNA translation kinetics inside the cell is, hence, crucial for a complete understanding of mRNA translation and its regulation.

In vivo super-resolution based single-molecule tracking techniques, have been successfully used to differentiate functional binding states of key macromolecules (recently reviewed in e.g. ${ }^{5}$ ). Initial applications of this approach has mostly been limited to the identification of cellular localizations of molecules in different functional states, whereas the dynamics of the processes has been out of reach due to limited photostability of the fluorescent proteins which have dominated the field of fluorescence microscopy. The development of new approaches to label biomolecules of interest, exploiting the brightness and photostability of small organic dyes, has opened up new possibilities for live-cell kinetics studies. Such approaches include, e.g., tracking of single dye-labeled tRNAs for translation kinetics measurements ${ }^{6}$, and measurements of spatiotemporal dynamics and translational activity of individual mRNAs ${ }^{7-11}$. The latter was accomplished by introducing arrays of labeling sites on both mRNA and nascent peptides, hence achieving multiple-fluorophore labeling of active translation sites.

In the present work, we introduce a system to directly study in vivo kinetics of translation initiation and elongation. We demonstrate that we can readily detect subtle changes in translation initiation kinetics as a response to antibiotics treatment or due to modifications in the ribosome itself. Our results further shed light on long-standing questions regarding how the bacterial ribosome finds the correct translation start site, and how polycistronic mRNAs are translated in the bacterial cell. 


\section{RESULTS}

\section{Construction of strains for ribosome labeling}

To track 30 S and 50 S ribosomal subunits in live Escherichia coli we constructed strains in which the gene for HaloTag ${ }^{12}$ was inserted at the C-terminus of the chromosomal locus for ribosomal proteins L1, L9, L19, L25, or S2 (Fig. 1A). Strains expressing L9-HaloTag and S2HaloTag showed minor negative effect on cell growth, cells in which L25 was mutated showed slower growth, while strains expressing L19-HaloTag or L1HaloTag showed pronounced growth defects (Fig. S1A).

Labeling of ribosomes by means of ribosomal proteins fused to the HaloTag provides a tool for tracking of the total pool of ribosomal subunits present in cells. However, this approach lacks discriminative power when only a subpopulation of ribosomes with specific

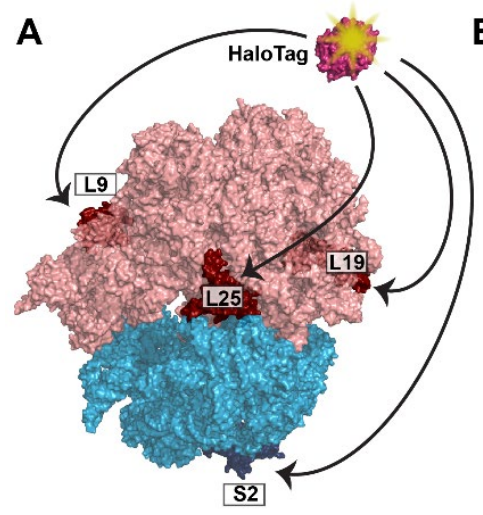

B ${ }_{\text {MS2CP-HaloTag }}$

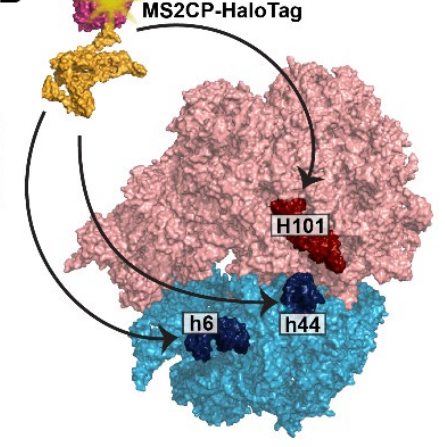

C

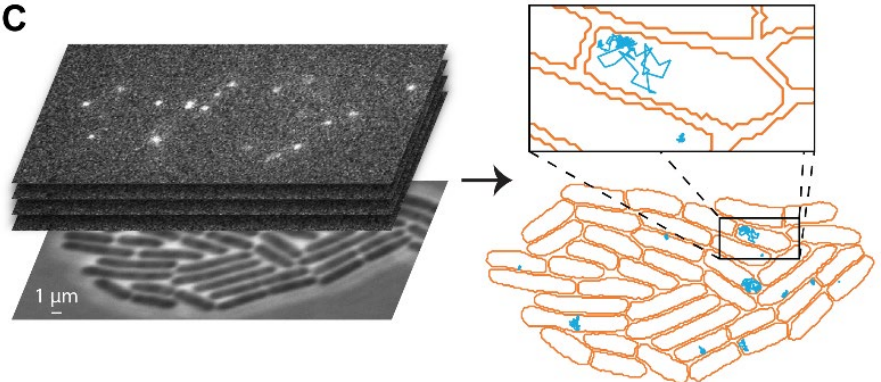

Fig. 1. Single-molecule tracking of ribosomal subunits. A. Site-specific labeling of ribosomal subunits was achieved by genetically fusing the HaloTag protein to a ribosomal protein (L9, L19, or L25 of the 50S subunit, and S2 of the 30S subunit). B. Site-specific labelling of only a subpopulation of ribosomal subunits was achieved by introducing the MS2 RNA aptamer into a surface exposed helix of ribosomal RNA (23S rRNA H101, 16S rRNA h6 or h44), to which a coexpressed MS2CP-HaloTag fusion protein binds. C. Fluorescence time-lapse movies of cells were acquired, and diffusion trajectories of labeled ribosomal subunits were automatically detected in cells segmented based on phasecontrast images.

properties is of interest. In several studies it has been shown that mutated ribosomes with desirable modifications can be isolated by introducing an MS2 RNA aptamer into the rRNA, which is then used for affinity purification by coupling to the strongly interacting MS2 coat protein $(\mathrm{MS2CP})^{13-15}$. Hence, to be able to track only a subpopulation of ribosomes, we inserted the MS2 RNA aptamer in different surface-exposed helices of the 16S rRNA or 23S rRNA (Fig. 1B) of an rrnB operon copy located on a plasmid. To test whether the insertion interferes with the activity 
of ribosomes, we introduced the different plasmids with the modified $r r n B$ operons into the $E$. coli SQ171 strain, which lacks all chromosomal rRNA operons. We found that extension of h6 of the 16S rRNA did not affect the growth rate of the cells, while cells with modified 16S rRNA h44 and $23 \mathrm{~S}$ rRNA H101 showed significant growth defects (Fig. S1B).

\section{Tracking of single fluorescent 305 and 50 s subunits in live cells}

For single-molecule tracking experiments we selected the L9-HaloTag and S2-HaloTag strains, as well as a strain containing both the plasmid for expression of h6-modified 16S rRNA and a plasmid encoding the MS2CP-HaloTag fusion. To fluorescence label the molecules fused to the HaloTag, cells in exponential growth phase were incubated with the JF549 HaloTag ligand which penetrates the cells and rapidly and specifically forms a covalent bond with the HaloTag protein ${ }^{16}$. After extensive washing, the cells were sparsely spread on an agarose pad, grown to mini colonies, and imaged at $37^{\circ} \mathrm{C}$ (Fig. 1C). For all strains tested, we observe that practically all ( 99\%) cells continued growth on the agarose pad and contained fluorescently labeled molecules. Labeled molecules were stable for several hours and were homogeneously distributed within mini colonies produced from each single labeled cell over time as a result of cell division (Fig. S2). Mini colonies were imaged using stroboscopic laser illumination with $3 \mathrm{~ms}$ laser pulses per $30 \mathrm{~ms}$ camera exposure frame. The resulting microscopy data obtained from more than 100 individual colonies were processed using a previously developed pipeline for automatic cell segmentation, fluorescent molecules detection, and building of single-molecule diffusion trajectories ${ }^{6}$ (Fig. 1C). The apparent average diffusion coefficient for the HaloTag molecules was very similar in all the selected strains (Fig. S3A) and consistent with the expected diffusion for ribosomal particles ${ }^{17-18}$, i.e. around $0.1 \mu \mathrm{m}^{2} / \mathrm{s}$. As a control, we also tracked free labeled HaloTag proteins in the genetic background strain, as well as MS2CP-HaloTag in cells expressing the $r r n B$ operon without insertion of the MS2 aptamer. In both these cases, the apparent average diffusion coefficient of the labeled molecules was approximately two orders of magnitude higher (Fig. S3B), matching what was previously reported for freely diffusing fluorescent proteins ${ }^{19}$. Hence, the HaloTag fusion to ribosomal proteins allow specific labeling of ribosomal subunits, whereas the presence of the MS2 aptamer in 16S rRNA allows specific labeling of a subpopulation of 30S for in vivo tracking. 


\section{HMM analysis of diffusion trajectories}

The high brightness and stability of the JF549 dye allowed us to track ribosomal subunits up to hundreds of frames (Fig. S4), which lead to the visual observation that the ribosomal particles do not diffuse homogeneously over time, but rather persist in different long-lived relatively discrete diffusional states (Supplementary Movie S1).

To obtain quantitative information on diffusion states and the transitions between these, we analyzed the diffusion trajectories using a Hidden Markov Modeling (HMM) approach20 previously used to extract tRNA-ribosome binding kinetics ${ }^{6}$. In this approach, all trajectories are fitted to a pre-set number of diffusion states, using the respective diffusion coefficients $(D)$ and the transition frequencies between these states as fitting parameters. Based on the results from previous single-molecule tracking of ribosomal subunits labeled with fluorescent proteins ${ }^{17-18}$, we expected that the HMM analysis should be able to distinguish freely diffusing ribosomal subunits from translating subunits bound to mRNA. However, we also anticipated that the 'bound' state, in particular, might not be well defined, but rather consist of a continuum of diffusion states representing everything from a comparably rapidly diffusing single $30 \mathrm{~S}$ or $70 \mathrm{~S}$ bound to an mRNA, to slowly diffusing polysomes, perhaps also tethered to the chromosome or bound to translocons in the cell membrane ${ }^{17,21-22}$. Hence, the diffusion trajectories were fitted to models with 2-8 discrete diffusion states. The average diffusion coefficient distributions are very similar between independent experiments (Fig. S5), and for different experimental replicas we observe good overall reproducibility in the diffusion states predicted by HMM (Tables S1-S3). To achieve convergence in the kinetics data, however, the results for all experiments below are presented from cumulated data from several independent experiments (2-5), with standard errors estimated by bootstrapping in the HMM fitting procedure.

For both the $30 \mathrm{~S}$ and $50 \mathrm{~S}$ tracking data, independent of model size, we find the largest fraction of ribosomal subunits diffusing at 0.03-0.06 $\mu \mathrm{m}^{2} / \mathrm{s}$, and a smaller fraction at around $0.4 \mu \mathrm{m}^{2} / \mathrm{s}$ (Fig. S6, Tables S4-S6). In models with more HMM states, the slower state is divided into several states, in agreement with our expectation of a loosely defined 'bound' state. Further, with model sizes of 6 or more states, low populated states $(<0.5 \%)$ at unphysiological ribosome diffusion 
rates $>3 \mu \mathrm{m}^{2} / \mathrm{s}$ appear. Based on our previous analysis of simulated fluorescence microscopy ${ }^{6}$, we tentatively assign these very rapid diffusion states as tracking artefacts, possibly in combination with a very small fraction of dissociated HaloTag protein.

\section{Diffusion of free ribosomal subunits}

Efficient initiation of translation for the majority of the $E$. coli genes depends on the presence of the Shine-Dalgarno (SD) motif, with a consensus sequence GGAGG located upstream of the AUG start codon. The SD motif facilitates recruitment of the $30 \mathrm{~S}$ subunit via RNA-RNA base-pairing with the corresponding CCUCC motif at the $3^{\prime}$ terminus of 16S rRNA, referred to as the anti-ShineDalgarno sequence $(A S D)^{23}$. Alternations in the ASD have been shown to render 30 s subunits unable to efficiently initiate translation of endogenous mRNAs, while mRNAs with the corresponding modifications in the SD motif were specifically translated only by such mutated, orthogonal, subunits ${ }^{24-25}$. Hence, to specifically study the diffusion of unbound 30 s subunits, we introduced the MS2 RNA aptamer into h6 of the orthogonal 30S (0-30S) developed in the Chin $\mathrm{lab}^{25}$, and co-expressed these with the MS2CP-HaloTag fusion.

We noticed that expression of O-30S subunits was moderately toxic for the $E$. coli cells, and that a C1400U mutation in the 16S rRNA of the 0-30S appeared frequently, which alleviates this toxicity. The $\mathrm{C} 1400$ residue in $16 \mathrm{~S}$ rRNS residue lies at the center of the decoding site of $16 \mathrm{~S}$ rRNA, and other mutations of C1400 in ASD-altered ribosomes greatly impact the overall activity of the ribosomes for both endogenous mRNAs and orthogonal mRNAs ${ }^{26}$. Hence, it is likely that 0-30S subunits are still significantly involved in translation of endogenous genes, perturbing the cell proteome, and that the $\mathrm{C} 1400 \mathrm{U}$ mutation reduces this activity ${ }^{27-28}$.

We tracked both 0-30S and 0-30S-U1400 and compared their diffusion with that of normal 30S subunits. The average diffusion of $0-30 \mathrm{~S}$ and $0-30 \mathrm{~S}-\mathrm{U} 1400$ was found to be $0.4 \mu \mathrm{m}^{2} / \mathrm{s}$ and 0.5 $\mu \mathrm{m}^{2} / \mathrm{s}$, respectively (Fig. 2A). This is significantly higher than $0.1 \mu \mathrm{m}^{2} / \mathrm{s}$ observed for $30 \mathrm{~S}$ subunits with unaltered ASD (Fig. 2A), thus suggesting that, in deed, both types of O-30S bind mRNA to less extent. From the HMM analysis of 0-30S trajectories we find that, for all model sizes, the occupancy (steady-state fraction) in the fast $\left(0.25-0.70 \mu \mathrm{m}^{2} / \mathrm{s}\right)$ diffusion states increases dramatically, to $\sim 50 \%$ and $\sim 80 \%$ for O-30S and O-30S-U1400, respectively, compared to $<10 \%$ 
for ribosomes with unaltered ASD (Fig. 2B and Tables S6-S8). Hence, a threshold for diffusion of $30 \mathrm{~S}$ subunits at $0.25 \mathrm{\mu m}^{2} / \mathrm{s}$ would enable us to separate freely diffusing $30 \mathrm{~S}$ from $30 \mathrm{~S}$ participating in translation. The difference in diffusion for 0-30S and 0-30S-U1400, further suggests that $0-30 S$ subunits partially retain their ability to bind and translate endogenous mRNAs, in agreement with results of ribosomal profiling for orthogonal ribosomes ${ }^{28}$, and that mutation of $U 1400$ dramatically reduces their affinity to mRNA.
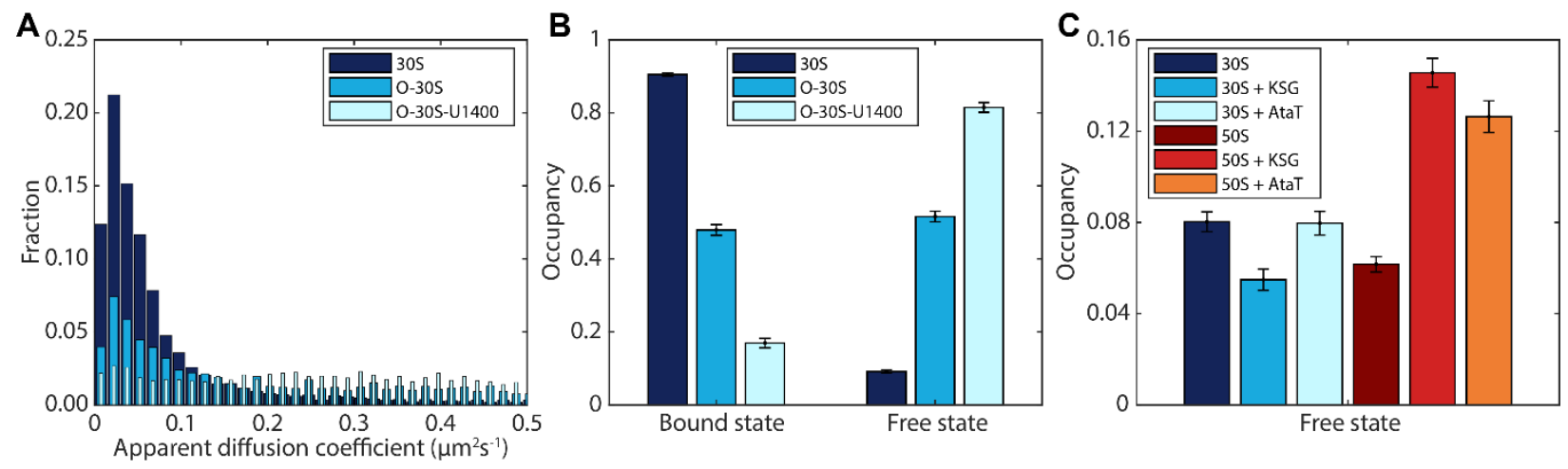

Fig. 2. Distinction between freely diffusing and mRNA-bound ribosomal subunits A. Distribution of apparent diffusion coefficient, estimated from mean-squared-displacement analysis of diffusion trajectory segments of 7 frames. B. HMM-estimated occupancy (steady-state fraction) of 30S subunits in mRNA-bound or freely diffusing state. C. HMM-estimated occupancy of $30 \mathrm{~S}$ and $50 \mathrm{~S}$ subunits in the freely diffusing state in presence or absence of $50 \mathrm{\mu g} / \mathrm{ml} \mathrm{KSG}$ or the AtaT toxin. Panels B and C show coarse-grained HMM results from a 6-state model, using $0.25 \mu \mathrm{m}^{2} / \mathrm{s}$ as cutoff between bound and free subunits. The complete results for all model sizes (2-8) are shown in Tables S6-S8. Error bars represent bootstrap estimates of standard errors.

To further validate the threshold, and to investigate whether the same threshold can be used for 50 S subunits, we tracked $30 \mathrm{~S}$ and $50 \mathrm{~S}$ in cells treated by kasugamycin (KSG), an aminoglycoside antibiotic that specifically target translation initiation ${ }^{29}$. It has been shown in vitro that KSG moderately affects the binding of $30 \mathrm{~S}$ subunit to an mRNA, but strongly inhibits its affinity to initiator $\mathrm{fMet}^{-\mathrm{tRNA}} \mathrm{fMet}^{30}$. Thus, KSG should prevent the formation of the $70 \mathrm{~S}$ initiation complex, and, in particular, the arrival of $50 \mathrm{~S}$ is expected to be delayed. E. coli cells containing either L9HaloTag or S2-HaloTag were grown on an agarose pad for 2 hours, after which the pads were soaked in media containing $50 \mu \mathrm{g} / \mathrm{ml}$ KSG (0.2 times MIC, at which cells show 1.5 times longer doubling times). From the HMM analysis of diffusion trajectories in cells subjected to KSG treatment, we observe that the occupancy in diffusion states in the interval of $0.25-0.70 \mu \mathrm{m}^{2} / \mathrm{s}$ ('Free state') increases from $6 \%$ to $15 \%$ for 50S, whereas the free state occupancy of 30 S instead decrease slightly (from $8 \%$ to 6\%, Fig. 2C, Tables S9-S10). The difference in proportion of free $30 \mathrm{~S}$ and $50 \mathrm{~S}$ in KSG treated cells, compared to the equal proportion in untreated cells (Fig. 2C), 
hence shows that our analysis is capable of distinguishing free 305 subunits from 305 subunits bound to an mRNA awaiting 50S joining. When cells were treated with higher KSG concentration $(500 \mu \mathrm{g} / \mathrm{ml}, 2 \mathrm{X} \mathrm{MIC})$, the diffusion distribution for both $30 \mathrm{~S}$ and $50 \mathrm{~S}$ particles changed radically, with $\sim 50 \%$ occupancy in the freely diffusive state (Tables S11-S12).

To corroborate the result from KSG treatment even further, we performed a complementary experiment using the AtaT toxin. In this experiment, strains encoding S2-HaloTag and L9-HaloTag were transformed with a plasmid for low level of expression of AtaT. AtaT is a toxin from the recently characterized toxin-antitoxin complex, AtaRT, which specifically modifies Met-tRNA ${ }^{\text {fMet }}$ by acetylating the free amino group of the methionine ${ }^{31}$. This modification prevents the interaction between tRNA ${ }^{\text {fMet }}$ and IF2 which leads to inefficient formation of the 70S initiation complex ${ }^{31}$. Effectively, expression of AtaT toxin should decrease the concentration of available initiator fMet-tRNA ${ }^{\mathrm{fMet}}$, slowing down the final steps of translation initiation, including $50 \mathrm{~S}$ subunit joining.

The results from tracking $30 \mathrm{~S}$ and $50 \mathrm{~S}$ subunits in AtaT producing cells were, as expected, very similar to what was observed in cells treated with $50 \mu \mathrm{g} / \mathrm{ml} \mathrm{KSG}$, showing an increase in the proportion of free 505 subunits whereas no significant effect was found on the 30S subunits (Fig. 2C, Tables S13-S14). Importantly, the experiments with KSG and AtaT treatment show that once the $30 \mathrm{~S}$ subunit established interactions with an initiation site, it is unlikely to leave it and instead awaits 505 arrival.

\section{Kinetics of translation elongation}

In addition to the occupancy in each diffusion state, the HMM analysis also provides the estimated transition frequencies between these states, or conversely, the average dwell-time in each state. To study specifically the transitions between free subunits and mRNA-bound subunits, we coarse-grained the HMM models of each size obtained for 50 S subunits (L9-HaloTag) and 30 S subunits (S2-HaloTag and h6-MS2-HaloTag) into three states in accordance with the control experiments presented in the previous section: mRNA bound ribosomal subunits at $D<$ $0.25 \mu \mathrm{m}^{2} / \mathrm{s}$, freely diffusing subunits with $D$ in the interval of $0.25-1.5 \mu \mathrm{m}^{2} / \mathrm{s}$, and a third state at $D>1.5 \mu \mathrm{m}^{2} / \mathrm{s}$ in which tracking artifacts detected in larger models appear. Both the occupancy 
and the dwell-time in the coarse-grained functional states converge well for both $30 \mathrm{~S}$ and $50 \mathrm{~S}$ subunits with increasing model size (Fig. 3A-B). For simplicity and clarity, in the following discussions, we thus present only coarse-grained numbers from the 6-state models. Whereas absolute numbers are slightly different between different model sizes, our conclusions do not depend on exact model size $\geq 4$ states.

To investigate the kinetics of translation inside rapidly growing $E$. coli cells, we first compare the steady-state fraction of 305 and 505 subunits in the free or mRNA-bound diffusion states. For the 50 subunit, we find that $94 \%$ are at any given moment engaged in translation. The corresponding value for the $30 \mathrm{~S}$ subunit is $92 \%$ or $91 \%$ for S2 or h6 labeling respectively (Fig. $3 \mathrm{C}$ ). Hence, the fraction of free subunits, $6-9 \%$, is somewhat lower than what has previously been reported using other methods (i.e. $15 \%{ }^{32}$. However, it has also been estimated that precursors of $30 \mathrm{~S}$ and $50 \mathrm{~S}$ subunits comprise approximately $10 \%$ of all subunit particles ${ }^{33}$. Since in our experiments, data were acquired 2 hours after labeling, and we see no or negligible exchange of the ribosomal proteins and MS2CP-HaloTag between different subunits, we most probably track only the diffusion of fully processed particles. Hence, from our analysis we estimate that $\geq 90 \%$ of the mature fraction of both subunits are actively translating at any given moment.

A

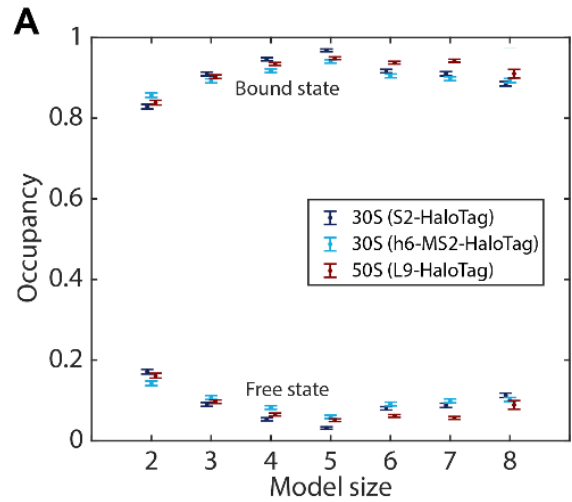

C

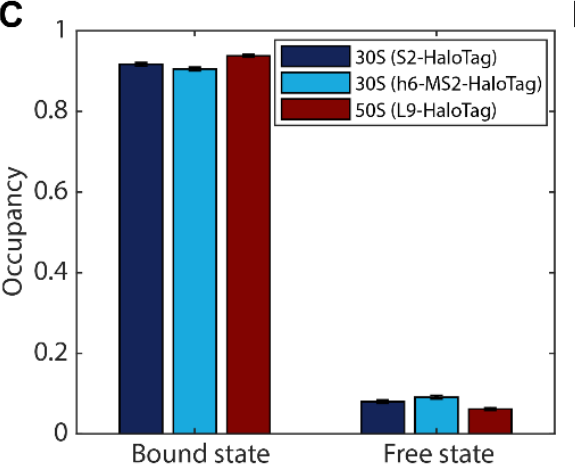

B
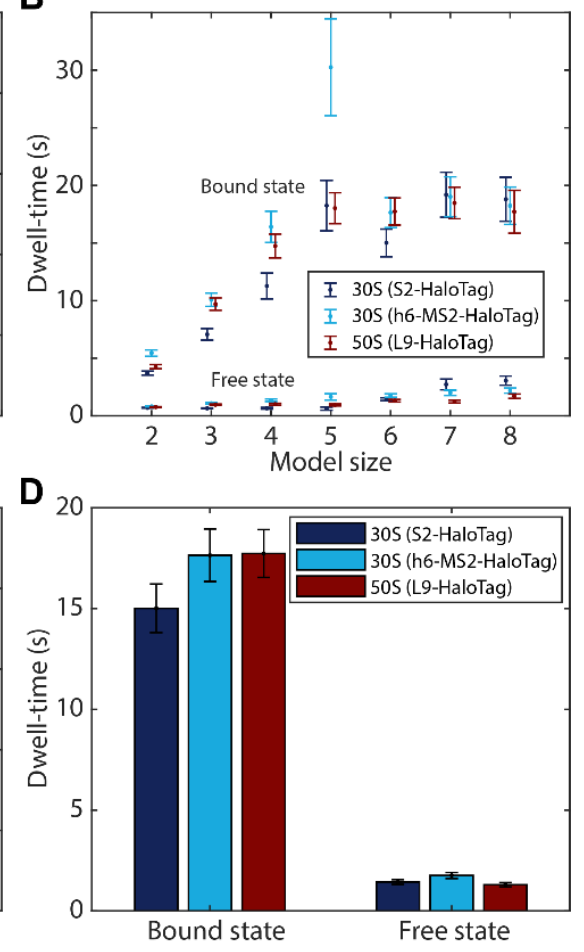

Fig. 3. Translation kinetics from coarse-grained HMMresults. A-B. Estimated occupancy and dwell-time of ribosomal subunits in the mRNA-bound state or the freely diffusive state for different HMM fitting model sizes. Full models (Tables S4S6) were coarse-grained into three states: mRNA-bound, free, and artifacts, using

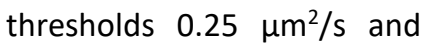
$1.5 \mu \mathrm{m}^{2} / \mathrm{s}$. C-D. Bar plots of model size 6 data as in panels A-B. Error bars represent bootstrap estimates of standard errors. 
From the HMM estimated transition frequencies of the ribosomal subunits between the diffusion states, we find that the $50 \mathrm{~S}$ subunits spend, on average, $18+/-1 \mathrm{~s}$ bound to an mRNA, whereas the time spent freely diffusing between binding events is on average $1.3 \mathrm{~s}+/-0.1 \mathrm{~s}$ (Fig. 3D). For the $30 \mathrm{~S}$ subunit, the corresponding numbers are $15+/-1 \mathrm{~s}$ and $1.4 \mathrm{~s}+/-0.1 \mathrm{~s}$, or $18+/-1 \mathrm{~s}$ and $1.8 s+/-0.2 s$ depending on the labeling strategy (Fig. 3D). Considering that elongating ribosomes has been estimated to proceed at a speed of 16-17 amino acids per second on average under similar growth conditions, i.e. 2 doublings $/ \mathrm{h}^{34-35}$, our estimates for the dwell-time in the slow diffusion state, $15-18 \mathrm{~s}$, are in a good agreement with the time required to translate one 'typical' E. coli protein with a length of $\sim 250$ amino acids ${ }^{36}$ which would take $15-16 \mathrm{~s}$.

The fact that the slow diffusion state dwell-time is the same for both subunits (Fig. 3D), have further interesting consequences. More than $50 \%$ of $E$. coli genes are organized in operons which are transcribed into polycistronic transcripts ${ }^{37}$. Polycistronic organization of the transcriptome was shown to be important for translational coupling of genes for which the exact stoichiometry is required for correct operation ${ }^{38-40}$. Such coupling has been suggested to occur through either or both of two hypothetical mechanisms ${ }^{41}$. In the first case, translation of a downstream gene by a ribosome only occurs when another ribosome is translating the upstream gene, thereby unwinding a stable mRNA structure, which would otherwise block the ribosome binding site of the downstream gene ${ }^{41}$. In the second scenario, initiation on the downstream gene occurs by the very same $30 S^{42}$ or $70 S^{43}$ which translated the upstream gene, without dissociation from the mRNA. Our estimates for the dwell-time in the mRNA bound diffusion state of both the small and the large ribosomal subunit (Fig. 3D), strongly suggest that, in the majority of mRNA binding events, ribosomes translate only one gene at a time. Further, since we see no apparent difference in dwell-times between the small and the large subunits (Fig. 3D), independent of model size (Fig. 3B), both subunits are likely to dissociate from an mRNA after translation termination.

\section{Selection of initiation sites by 30 subunits}

During translation initiation, the 305 subunit is required to rapidly scan the transcriptome in order to identify a correct start codon in a vast pool of potential sites. In eukaryotes, the identification of initiation sites on mRNA by the small ribosomal subunit is not direct, but requires initiation 
factors, specific mRNA structures, and mRNA scanning ${ }^{44}$. In E. coli, however, initiation sites on mRNAs can be recognized by $30 \mathrm{~S}$ alone ${ }^{45}$, and since the majority of bacterial mRNAs are polycistronic, initiation sites should be searched throughout the entire transcripts, and not only by the $5^{\prime}$ end. In certain cases, diffusion along an mRNA has been shown to promote translation initiation $^{42}$, but it is generally assumed that the 305 subunit binds directly or in very close proximity to the initiation site. Base pairing between SD and ASD should, in principle, direct the $30 S$ to the start codon. However, a recent study employing ribosomes with a modified ASD sequence, provided strong evidence that the SD-ASD interaction is far from enough for efficient translation, and that initiation sites are rather hardwired by other mRNA features ${ }^{28}$. It has also been shown that the initial association of $30 \mathrm{~S}$ with mRNAs depends on secondary structures by the initiation site, independent of the SD motif and start codon, which are monitored in subsequent steps ${ }^{46-47}$. Yet, it is still debated to what extent initiation relies on SD-ASD interaction in E. coli and how widespread this mechanism of modulation of translation initiation is in other bacteria $^{48-49}$.

In the above experiments, which helped us to establish the threshold for separation of free and mRNA-bound 30 S subunits, we noticed that the previously developed $0-30 S^{25}$ appear to be significantly involved in binding of endogenous mRNAs (Fig. 2A-B). Besides a mutated ASD sequence, these ribosomes carry the additional 16S rRNA substitutions G722C and U723A located in the vicinity of the helix formed by SD and ASD, as well as a C1192U mutation which confers spectinomycin resistance ${ }^{50}$. To investigate the contribution from the ASD sequence alone, as well as these additional rRNA mutations, we analyzed the occupancy and dwell-times of several modified 305 subunits in the freely diffusing state and the mRNA-bound state using the coarsegrained HMM-analysis as described above (Tables S15-S18). To minimize the effect of toxicity associated with expression of ASD-modified ribosomes, these experiments were performed with new constructs, with only low-level expression of the mutated rRNA operons.

For 305 subunits in which only the ASD sequence was mutated (O-ASD), a substantial fraction (69\%) was still found to be in the mRNA-bound state at any given moment, although the corresponding O-SD is not present in the cells. Incorporation of the G722C-U723A mutations (OASD-C722-A723) and the additional C1192U mutation (O-30S) decrease this fraction further, to 
$57 \%$ and $53 \%$ respectively, whereas the additional C1400U mutation (O-30S-U1400) finally brings the bound-state occupancy down to a mere $23 \%$ (Fig. 4A).

A

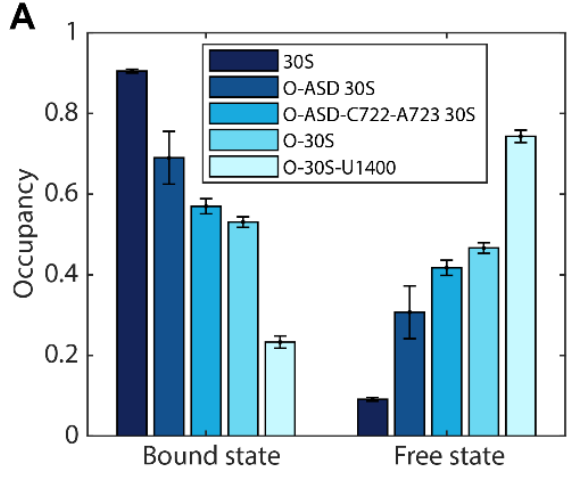

B

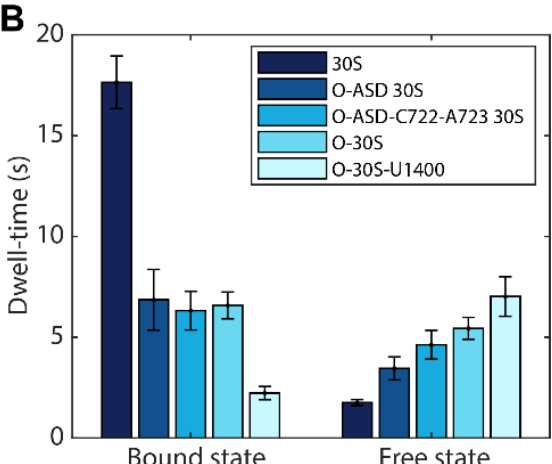

Fig. 4. Translation kinetics of modified $30 S$ subunits. Estimated occupancy (A) and dwell-time (B) of tracked $30 \mathrm{~S}$ subunit variants in the mRNAbound state or the freely diffusive state. The data shows the coarse-grained results from HMM-fitted model size 6 . Results for all model sizes (2-8), are shown in Table $\mathrm{S} 6$ and Tables S15-S18. Error bars represent bootstrap estimates of standard errors.

From the HMM estimated transition frequencies between the free and mRNA-bound diffusion states, we find that O-ASD, O-ASD-C722-A723, and O-30S spend, on average, approximately the same $6.5 \mathrm{~s}$ in the mRNA-bound state, whereas the time spent freely diffusing between binding events is between $3.5 \mathrm{~s}$ and $5.5 \mathrm{~s}$ (Fig. 4B). The relatively long-lived bound state for these mutants suggests their involvement in translation, although it appears to be significantly shorter than what we have calculated for unmodified ribosomes (16-18 s, Fig. 3D and 4B). Selective ribosome profiling on ribosomes with altered ASD sequences has revealed vast differences in translation efficiencies for different genes between wt ribosomes and ASD mutant ribosomes ${ }^{28}$. Based on published data of the relative ribosomal occupancies on individual genes for the O-ASD mutant ${ }^{28}$, we estimate the average length of polypeptides translated by O-ASD ribosomes to be $\sim 150$ amino acids (see Methods). Indeed, this is much shorter than the 'average' protein translated by wt ribosomes, $\sim 250$ amino acids ${ }^{36}$. Moreover, close investigation of ribosome density profiles for individual genes revealed that in a substantial number of instances, initiation occurs at alternative start codons in which orthogonal ribosomes are likely to translate only short nonsense ORFs. Hence, we conclude that the observed binding events in O-ASD-mutant tracking likely represent active translation, albeit of, on average, shorter sequences.

On average it takes only twice longer for O-ASD ribosomes to find initiation sites relative to unmodified 30S (3.5 s +/- 0.6 s vs 1.8 s +/- 0.2 s, Fig. 4B). Incorporation of mutations G722CU723A and C1192U lead to slightly longer time needed for initiation (4.6 s +/- $0.7 \mathrm{~s}$ and $5.4 \mathrm{~s}+$ /- 
$0.5 \mathrm{~s}$, respectively), probably reflecting less affinity to mRNAs. These three $30 \mathrm{~s}$ variants, however, are likely to have similar preferences in what genes to translate, given the similar average dwelltimes in the bound state (Fig. 4B). Finally, the most severely affected 30S mutant, with the additional C1400U mutation in the decoding center, shows dramatically different transition frequencies between free and mRNA-bound diffusion states, with a long-lived free state (7 s +/$1 \mathrm{~s}$ ), interrupted only by relatively short binding events for $2.2 \mathrm{~s}+/-0.3 \mathrm{~s}$ (Fig. 4B). Hence, these orthogonal subunits seem not to translate endogenous mRNAs to basically any extent at all.

\section{CONCLUSIONS}

We have presented a comprehensive analysis of diffusional behavior of $E$. coli ribosomes and developed a toolset to study global kinetics of mRNA translation directly in living cells. Using this approach, we found that both $E$. coli ribosomal subunits are likely to dissociate from the mRNA after translation termination. Hence, we see no evidence of widespread re-initiation on downstream ORFs by $30 \mathrm{~S}^{42}$ or $70 \mathrm{~S}^{43}$ remaining bound to the mRNA.

We further examined the importance of the SD-ASD interaction for translation initiation, and show that it has only a modest impact on the overall initiation efficiency. Rather, other features of mRNAs play major roles for regulation of protein expression. It will, thus, be interesting, to further investigate the co-evolution of the transcriptome with ribosomes, and look for specific features of the ribosomes which enable specific interactions with mRNAs. Such findings would significantly help in the development of artificial ribosomes with higher level of orthogonality for bioengineering applications.

Finally, our single-molecule tracking method allows quick evaluation of the translational state of cells, and detection of even subtle changes in state occupancies or state transition rates. After preparation of cells with labeled ribosomal subunits, this information is readily accessible within minutes without any downstream processing after data acquisition, thus minimizing the risk of artefacts. The method further allows for testing of multiple conditions and to introduce changes over the course of the experiment. Hence, the method opens up new possibilities to investigate how bacteria rapidly adapt to changing conditions, such as antibiotics treatment, and regulate their protein synthesis machinery for optimal recourse allocation. 


\section{ACKNOWLEDGMENTS}

The authors would like to thank the laboratory of Luke Lavis for providing the JF549-HaloTag dye. This work was supported by The Swedish Research Council (M.J. 2015-04111, 2016-06264, 201903714; J.E. and M.J. 2016-06213), the Knut and Alice Wallenberg Foundation (J.E.), and the Carl Trygger Foundation (M.J. 15:243).

\section{METHODS}

\section{Construction of bacterial strains and growth conditions}

For cloning purposes all PCRs were done using Q5 high-fidelity DNA polymerase (NEB) and Gibson Assembly was performed using NEBuilder HiFi DNA assembly master mix (NEB) according to manufacturer's protocols. Strains expressing L1-, L9-, L25, and S2-HaloTag were constructed using $\lambda$ Red assisted recombineering ${ }^{51}$. First, the pKD4 plasmid (Addgene \#45605) was amplified by inverse PCR using primers pKD4_F and pKD4_R (Table 1) and Gibson-assembled with the HaloTag gene amplified with primers HaloTag_F and HaloTag_R (Table 1) and pFA6a-HaloTagKanMX6 (Addgene \#87029) as a template. Next, the HaloTag-KmR containing fragments were amplified from the resulted pKD4-HaloTag plasmid with pairs of primers X-Halotag-F and XHalotag- $R$, where $X$ is the ribosomal protein to be mutated (Table 1). The amplified fragments were used for recombineering to insert HaloTag-KmR cassettes in the genome of E. coli MG1655 at the C-terminus of each selected gene ${ }^{51}$.

Insertion of the MS2 aptamer into different positions of the $r r n B$ operon encoded in the pAM552 plasmid (a gift from A. Mankin lab, as in Addgene \#154131) was performed by inverse PCR using primers Y-MS2-F and Y-MS2-R (Table 1), where $Y$ designate the ribosomal helix which was modified, followed by re-circularization through PNK phosphorylation of PCR product ends and ligation by T4 ligase (NEB). Similarly, to create a plasmid for tracking of ribosomes with mutated ASD sequence, the MS2 aptamer insertion at h6 was performed by inverse PCR of the pSC101-Oribosome (a gift from J. Chin lab) using primers h6-MS2-F and h6-MS2-R (Table 1) and subsequent re-circularization resulting in plasmid pSC101-O-ribosome-h6-MS2. Selection of mutation 
C1400U in 16 rRNA in pSC101-O-ribosome-h6-MS2 was confirmed by Sanger sequencing of isolated plasmids from independent colonies showing faster growth on LB plates.

To create a plasmid in which only the ASD sequence is mutated and additional mutations (G722C, U723A, and C1192U) are not present, the fragment containing the corresponding positions was amplified using oligonucleotides Ribo600-F and Ribo1465-R (Table 1) from the pAM552 plasmid, and the PCR product was Gibson assembled with the fragment of the plasmid pSC101-Oribosome-h6-MS2 amplified using primers Ribo1465-F and Ribo600-R (Table 1). The resulting plasmid pSC101-oASD-h6-MS2, when transformed in E. coli MG1655, caused high level of toxicity and instability. Therefore, a set of plasmids with a weaker apFAB59 promoter from the BIOFAB collection $^{52}$ (p59) regulating expression of mutated ribosomal operon was created. First, the P1P2 promoter present in pSC101-O-ribosome-h6-MS2 was exchanged with the p59 promoter by inverse PCR using primers P59-rrnB-F and P-rrnB-R (Table 1), and subsequent re-circularization resulting in plasmid pSC101-P59-O-ribosome-h6-MS2. Next, a derivative of this plasmid in which only the ASD sequence is mutated was created using Gibson Assembly as explained above, yielding the plasmid pSC101-P59-oASD-h6-MS2. Finally, the plasmid pSC101-P59-oASD-C722A723-h6-MS2, in which 16S rRNA contains the mutated ASD sequence and substitutions G722C and U723A, was created by inverse PCR using pSC101-P59-OASD-h6-MS2 as a template and primers C722-A723-F and 721-R (Table 1), with subsequent re-circularization.

Several plasmids that allow expression of MS2CP-HaloTag were created. First, the lacUV5 promoter was inserted in the pCOLADuet vector (Novagen) by inverse PCR using primers PCOLAlac-F and pCOLA-lac-R (Table 1), followed by re-circularization by ligation which resulted in the pCOLA-lacUV5 plasmid. Then, the gene for single-chain tandem dimer of the high affinity version of MS2 coat protein and the gene for HaloTag were Gibson-assembled with the pCOLA-lacUV5 plasmid. Corresponding fragments were obtained by PCR amplification using pairs of primers: i) MS2CPd-F and MS2CPd-R for amplification of MS2CP; ii) HaloTag-F and HaloTag-R for amplification of HaloTag; iii) pCOLA-GA-F and pCOLA-GA-R for the linearized plasmid (Table 1). This resulted in in the pCOLA-lacUV5-MS2CP-HaloTag plasmid. Next, a fragment from the pCOLAlacUV5-MS2CP-HaloTag plasmid, containing the lacl gene, lacUV5 promoter and MS2CP-HaloTag fusion, was amplified with primers MS2CP-Halo-F (Table 1) and MS2CP-Halo-R (Table 1). This 
fragment was fused via Gibson Assembly with two fragments: i) a DNA fragment containing the p15 origin of replication and the gene for chloramphenicol acetyltransferase, and ii) the $r r n B$ terminator sequence. The first fragment was amplified using p15-Cam-F and p15-Cam-R (Table 1) from the plasmid pEVOL-pBpF (Addgene \#31190), and the second fragment was obtained from the $\mathrm{pBAD} /$ HisB vector (Invitrogen) by amplification using primers rrnBterm- $\mathrm{F}$ and rrnBterm-R (Table 1). The resulting plasmid is called p15-lacUV5-MS2CP-HaloTag. Finally, a derivative of this plasmid in which the lacl gene was removed and the lacUV5 promoter was swapped with a weak constitutive promoter apFAB124 from the BIOFAB collection ${ }^{52}$ was created by inverse PCR using primers p124-MS2-Halo-F and p124-MS2-Halo-R (Table 1) and subsequent re-circularization.

A plasmid pBAD30-ataT containing the gene for the AtaT toxin (a gift from S. Dubiley) from the AtaRT toxin-antitoxin system with a $P_{B A D}$ promoter regulating the expression ${ }^{31}$ was modified to reduce the expression level by substituting the strong AGGAGG Shine-Dalgarno to the weak TTCTCA sequence by inverse PCR using primers AtaT-F and AtaT-weakSD-R (Table 1) and subsequent re-circularization resulting in plasmid pBAD30-SD weak-AtaT.

Table 1. Oligonucleotides used for cloning.

\begin{tabular}{|c|c|}
\hline Name & Sequence, 5'-3' \\
\hline HaloTag-F & acggctgacatgggaattagatggcagaaatcggtactgg \\
\hline HaloTag-R & gatattcatatggaccatggetagccggaaatctcgagcgtcgacagc \\
\hline pKD4-F & cgetcgagatttccggetagccatggtccatatgaatatcctccttagt \\
\hline pKD4-R & ccagtaccgatttctgccatctaattcccatgtcagccgttaagtg \\
\hline L1-Halotag-F & gttgcagttgaccaggctggcctgagcgcttctgtaaacggcgcagaaatcggtactggc \\
\hline L1-Halotag-R & gggtaagattgtagacaaaatcaccgcccacgtaaaggcagtgtaggctggagctgcttc \\
\hline L9-Halotag-F & gaagtattcgcgaaagtgatcgtaaacgtagtagctgaaggcgcagaaatcggtactggc \\
\hline L9-Halotag-R & accaatggtcggcgtttttacgtctcgttgaataacgaaagtgtaggctggagctgcttc \\
\hline L19-Halotag-F & cgtactggtaaggctgctcgtatcaaagagcgtcttaacggcgcagaaatcggtactggc \\
\hline L19-Halotag-R & ggccagccettcttaacaggatgtcgcttaagcgaaatcagtgtaggctggagctgcttc \\
\hline L25-Halotag-F & tacaaaccgaagctgcagcacatcgacttcgttcgcgctggcgcagaaatcggtactggc \\
\hline L25-Halotag-R & accccgccggagcggggttttttacaacttattcagcaaagtgtaggctggagctgcttc \\
\hline S2-Halotag-F & ctggcttcccaggcggaagaaagcttcgtagaagctgagggcgcagaaatcggtactggc \\
\hline S2-Halotag-R & tctgcaactcgaactattttgggggagttatcaagccttagtgtaggctggagctgcttc \\
\hline h6-MS2-F & actagttttgatgaggatcacccatctttactagtcttctttgctgacgagtggcg \\
\hline h6-MS2-R & cttcttcctgttaccgttcgacttg \\
\hline
\end{tabular}




\begin{tabular}{|c|c|}
\hline h44-MS2-F & actagttttgatgaggatcacccatctttactagtggagggcgcttaccactttgtg \\
\hline h44-MS2-R & ggttaagctacctacttcttttgc \\
\hline H101-MS2-F & actagttttgatgaggatcacccatctttactagttgcgttgagctaaccggtac \\
\hline H101-MS2-R & tgcgettacacacccggecta \\
\hline Ribo1465-F & ccactttgtgattcatgactggggtgaag \\
\hline Ribo1456-R & cttcacccagtcatgaatcacaaagtgg \\
\hline Ribo600-F & gatgtgaaatccccgggctcaacctgggaactgcatctgatact \\
\hline Ribo600-R & tcagatgcagttcccaggttgageccggggat \\
\hline P59-rrnB-F & ttgacaattaatcatccggetcgatacttacagccatcccgegccgetgagaaaaagcga \\
\hline P-rrnB-R & gaggaaatttaaaataattttctgaccgcg \\
\hline C722-A723-F & caggcgaaggcggeccctgga \\
\hline $721-\mathrm{R}$ & cggtattcctccagatctctacgc \\
\hline pCOLA-lac-F & gtggaattgtgagcggataacaatttgctgccaccgctgagcaataa \\
\hline pCOLA-lac-R & acattatacgagccggaagcataaagtgtaaagcatttcctaatgcaggagtcgcataag \\
\hline MS2CPd-F & ttgtgagcggataacaatttctagcaggaggaattgatggcttct \\
\hline MS2CPd-R & aagccagtaccgatttctgcgcagaagggggatccgtaga \\
\hline HaloTag-F & tctacggatccccettctgcgcagaaatcggtactggetttcc \\
\hline HaloTag-R & tattgctcagcggtggcagcctagccggaaatctcgagcg \\
\hline pCOLA-GA-F & cgctcgagatttccggetaggctgccaccgetgagc \\
\hline pCOLA-GA-R & ccatcaattcctcctgctagaaattgttatccgctcacaattccac \\
\hline lacUV5-MS2CP-Halo-F & taagcttggtacccaaccaagctcactgccegctttcc \\
\hline lacUV5-MS2CP-Halo-R & caaaacagccaagcaagcttctagccggaaatctcgagcg \\
\hline rrnBterm-F & cgctcgagatttccggctagaagcttgcttggctgttttgg \\
\hline rrnBterm-R & agcaggcttttcgaatttggaacaaaagagtttgtagaaacgcaaaaagg \\
\hline p15-Cam-F & tttctacaaactcttttgttccaaattcgaaaagcctgctcaac \\
\hline P15-Cam-R & ctggaaagcgggcagtgagcttggttgggtaccaagcttatcgatga \\
\hline p124-MS2-Halo-F & gaattcaaaagatctaaagaggagaaaggatctatggcttctaactttactcagttcgtt \\
\hline p124-MS2-Halo-R & atggctgtaagtattcgccgcaagggataaatgtcgagctgtcaaacatgagaattacaa \\
\hline AtaT-F & atggatgatctgacgatagagattc \\
\hline AtaT-weakSD-R & ttttaattgagaatgaattcgctagcccaaaaaaacg \\
\hline
\end{tabular}

\section{Calculation of doubling time}

Bacterial strains were grown on LB agar plates overnight at $37^{\circ} \mathrm{C}$. For each tested strain, six individual colonies were grown in $L B$ media until they reached $\mathrm{OD}_{600}=0.1-1$. The cells were then 
diluted to $\mathrm{OD}_{600}=0.0001$ in fresh $\mathrm{LB}$ and growth kinetics were recorded at $37^{\circ} \mathrm{C}$ in a 96 -well microplate (100 $\mu \mathrm{l} /$ well) using Spark $10 \mathrm{M}$ microplate reader (Tecan) with measurements of $\mathrm{OD}_{600}$ performed every $5 \mathrm{~min}$ after $1 \mathrm{~min}$ shaking. After subtraction of medium background, the minimal doubling time was calculated using a custom MATLAB script, which fits growth curves of cells with an exponential function and identifies $30 \mathrm{~min}$ intervals of maximal growth rate for each curve. The data from six independent wells were fitted separately and the results were averaged.

\section{HaloTag labeling and sample preparation}

E. coli cells containing HaloTag were grown on LB agar plates overnight at $37^{\circ} \mathrm{C}$. Several colonies were used to inoculate EZ Rich Defined Medium (RDM, Teknova) supplemented with 0.2\% glucose. Cells were grown at $37^{\circ} \mathrm{C}$ with shaking to $\mathrm{OD}=0.5-1$, harvested by centrifugation at 2000 g, resuspended in $150 \mu$ l of fresh RDM media, and JF549 HaloTag ligand (a gift from Lavis lab) was added to the final concentration of $0.5 \mu \mathrm{M}$. After incubation with the ligand for $30 \mathrm{~min}$ at $25^{\circ} \mathrm{C}$, cells were washed twice using M9 media supplemented with $0.2 \%$ glucose, then incubated in RDM media at $37^{\circ} \mathrm{C}$ with shaking for $60 \mathrm{~min}$ to facilitate the release of unbound ligand from cells. After incubation, cells were washed 3 times using M9 media supplemented with $0.2 \%$ glucose. Finally, cells were resuspended in $\mathrm{RDM}$ to $\mathrm{OD}_{600} \approx 0.003$ and sparsely spread onto an agarose pad prepared with RDM and 2\% agarose (SeaPlaque GTG Agarose, Lonza). The sample was placed on the microscope with a cage incubator maintaining temperature at $37 \pm 2{ }^{\circ} \mathrm{C}$. Cells were grown for 2 hours forming mini-colonies before image acquisition.

For KSG treatment experiments, cells were incubated on agarose pads at $37^{\circ} \mathrm{C}$ for 2 hours after which RDM media supplemented with $0.2 \%$ glucose and containing either $50 \mu \mathrm{g} / \mathrm{ml}$ or $500 \mu \mathrm{g} / \mathrm{ml}$ KSG was injected to the sample with growing mini-colonies. Imaging of cells was performed 60 min after injection of antibiotic. For experiment in which production of AtaT toxin was induced, E. coli MG1655 expressing S2- or L9-HaloTag and transformed with the pBAD30-SD weak-AtaT plasmid were grown on an agarose pad to form mini-colonies for 2 hours at $37^{\circ} \mathrm{C}$. Production of the toxin was induced by injection of RDM media supplemented with $0.2 \%$ glucose and $0.2 \%$ arabinose. Imaging was performed 70 min later. 
Each experimental dataset included 2-5 replicated microscopy experiments, each comprising 30-100 cell colonies (8-100 cells each). The data were found to be consistent in between different repetitions and were combined for analysis.

\section{Optical Setup}

An inverted microscope (Nikon Ti-E) in combination with a CFI Apo TIRF $100 \times 1.49$ NA objective (Nikon) was used. Bright-field and fluorescence images were acquired using an iXon 897 Ultra EMCCD camera (Andor) coupled to an additional $2.0 \times$ lens (Diagnostic Instruments DD20NLT). Phase contrast imaging was performed with an Infinity2-5 M camera (Lumenera). To track JF549 bound to HaloTag, a $553 \mathrm{~nm}$ laser (SLIM-553, Oxxius, 150mW) with a power density of $3 \mathrm{~kW} / \mathrm{cm}^{2}$ on the sample plane was used in stroboscopic illumination mode with 3 ms laser pulses per 30 ms camera exposures. The microscope was controlled using the $\mu$ Manager software package. Data acquisition from multiple positions was performed using custom $\mu$ Manager plugins.

\section{Single-particle tracking and analysis of trajectories}

Data analysis was performed using custom-made analysis pipelines written in MATLAB (available upon request). Single particle tracking was performed as described previously ${ }^{6}$. Briefly, boundaries of individual cells were extracted from phase contrast images using a previously developed algorithm ${ }^{53}$. Incorrectly segmented cells were manually removed and discarded from the subsequent analysis. Fluorescent spots were detected using the radial symmetry-based method $^{54}$. Refinement of spot positions and estimation of position uncertainties was performed using a symmetric Gaussian spot modelling and the maximum aposteriori fitting ${ }^{20}$. Twodimensional trajectory building was done using uTrack $^{55}$. Trajectories were built in cells starting from the time-point when only one spot remained in the cell in current and following frames, allowing gaps with 1 missing point. Spots with width (std) $>280 \mathrm{~nm}$, with amplitute $<50$ photons, or outside live cells (>3 pixels outside of boundaries) were excluded. Using a previously described HMM algorithm ${ }^{6,20}$ we analyzed diffusional properties of tracked molecules by fitting all trajectories with length of $\geq 20$ steps obtained for each experimental condition to a pre-set number of diffusion states. The algorithm makes explicit use of spot coordinates as well as localization uncertainties of each spot and handles missing data points ${ }^{20}$. Mean dwell times were 
calculated from the diagonal elements of the resulted HMM transition matrix, i.e., from the average probability of exiting a diffusive state, thus allowing the estimation of dwell times which are longer than the span of trajectories. To condense HMM models with many states to a coarse grained three-state model, the hidden states were classified as "mRNA bound", "free", and "unphysiological" using threshold values of $0.25 \mu \mathrm{m}^{2} / \mathrm{s}$ and $1.5 \mu \mathrm{m}^{2} / \mathrm{s}$.

For mean square displacement (MSD) analysis, trajectories were sliced into segments of 7 positions, and linear fits passing through the origin was made using the equation $M S D(t)=$ $2 n D \Delta \mathrm{t} \cdot \mathrm{t}$, where $t=1,2,3$ (first 3 points), $n=2$ is the dimension of the trajectories, $\Delta t=30 \mathrm{~ms}$ is the time step, and the apparent diffusion coefficient $D$ is the fitting parameter.

\section{Analysis of previously published ribosome profiling data}

The average length of polypeptides synthesized by O-ASD 30 S was calculated from the ribosome density fraction on the top hundred most translated genes (comprising about $2 / 3$ of all translation) in the O-ASD 30 S ribosome profiling data $\mathrm{in}^{28}$.

\section{REFERENCES}

1. Rodnina, M. V., The ribosome in action: Tuning of translational efficiency and protein folding. Protein Sci 2016, 25 (8), 1390-406.

2. Frank, J., The translation elongation cycle-capturing multiple states by cryo-electron microscopy. Philos Trans R Soc Lond B Biol Sci 2017, 372 (1716).

3. Kaledhonkar, S.; Fu, Z.; Caban, K.; Li, W.; Chen, B.; Sun, M.; Gonzalez, R. L., Jr.; Frank, J., Late steps in bacterial translation initiation visualized using time-resolved cryo-EM. Nature 2019, 570 (7761), 400-404.

4. Prabhakar, A.; Puglisi, E. V.; Puglisi, J. D., Single-Molecule Fluorescence Applied to Translation. Cold Spring Harb Perspect Biol 2019, 11 (1).

5. Elf, J.; Barkefors, I., Single-Molecule Kinetics in Living Cells. Annu Rev Biochem 2019, 88, 635659.

6. Volkov, I. L.; Linden, M.; Aguirre Rivera, J.; leong, K. W.; Metelev, M.; Elf, J.; Johansson, M., tRNA tracking for direct measurements of protein synthesis kinetics in live cells. Nat Chem Biol 2018, 14 (6), 618-626.

7. Morisaki, T.; Lyon, K.; DeLuca, K. F.; Deluca, J. G.; English, B. P.; Zhang, Z.; Lavis, L. D.; Grimm, J. B.; Viswanathan, S.; Looger, L. L.; Lionnet, T.; Stasevich, T. J., Real-time quantification of single RNA translation dynamics in living cells. Science 2016, 352 (6292), 1425-9. 
8. Pichon, X.; Bastide, A.; Safieddine, A.; Chouaib, R.; Samacoits, A.; Basyuk, E.; Peter, M.; Mueller, F.; Bertrand, E., Visualization of single endogenous polysomes reveals the dynamics of translation in live human cells. J Cell Biol 2016, 214 (6), 769-81.

9. Wang, C.; Han, B.; Zhou, R.; Zhuang, X., Real-Time Imaging of Translation on Single mRNA Transcripts in Live Cells. Cell 2016, 165 (4), 990-1001.

10. Wu, B.; Eliscovich, C.; Yoon, Y. J.; Singer, R. H., Translation dynamics of single mRNAs in live cells and neurons. Science 2016, 352 (6292), 1430-5.

11. Yan, X.; Hoek, T. A.; Vale, R. D.; Tanenbaum, M. E., Dynamics of Translation of Single mRNA Molecules In Vivo. Cell 2016, 165 (4), 976-89.

12. Los, G. V.; Encell, L. P.; McDougall, M. G.; Hartzell, D. D.; Karassina, N.; Zimprich, C.; Wood, M. G.; Learish, R.; Ohana, R. F.; Urh, M.; Simpson, D.; Mendez, J.; Zimmerman, K.; Otto, P.; Vidugiris, G.; Zhu, J.; Darzins, A.; Klaubert, D. H.; Bulleit, R. F.; Wood, K. V., HaloTag: a novel protein labeling technology for cell imaging and protein analysis. ACS Chem Biol 2008, 3 (6), 373-82.

13. Barrett, O. P.; Chin, J. W., Evolved orthogonal ribosome purification for in vitro characterization. Nucleic Acids Res 2010, 38 (8), 2682-91.

14. Youngman, E. M.; Green, R., Affinity purification of in vivo-assembled ribosomes for in vitro biochemical analysis. Methods 2005, 36 (3), 305-12.

15. Lowary, P. T.; Uhlenbeck, O. C., An RNA mutation that increases the affinity of an RNA-protein interaction. Nucleic Acids Res 1987, 15 (24), 10483-93.

16. Grimm, J. B.; English, B. P.; Chen, J.; Slaughter, J. P.; Zhang, Z.; Revyakin, A.; Patel, R.; Macklin, J. J.; Normanno, D.; Singer, R. H.; Lionnet, T.; Lavis, L. D., A general method to improve fluorophores for live-cell and single-molecule microscopy. Nat Methods 2015, 12 (3), 244-50, 3 p following 250.

17. Bakshi, S.; Siryaporn, A.; Goulian, M.; Weisshaar, J. C., Superresolution imaging of ribosomes and RNA polymerase in live Escherichia coli cells. Mol Microbiol 2012, 85 (1), 21-38.

18. Sanamrad, A.; Persson, F.; Lundius, E. G.; Fange, D.; Gynna, A. H.; Elf, J., Single-particle tracking reveals that free ribosomal subunits are not excluded from the Escherichia coli nucleoid. Proc Natl Acad Sci U S A 2014, 111 (31), 11413-8.

19. English, B. P.; Hauryliuk, V.; Sanamrad, A.; Tankov, S.; Dekker, N. H.; Elf, J., Single-molecule investigations of the stringent response machinery in living bacterial cells. Proc Natl Acad Sci U $S$ A 2011, 108 (31), E365-73.

20. Linden, M.; Curic, V.; Amselem, E.; Elf, J., Pointwise error estimates in localization microscopy. Nat Commun 2017, 8, 15115.

21. Kohler, R.; Mooney, R. A.; Mills, D. J.; Landick, R.; Cramer, P., Architecture of a transcribingtranslating expressome. Science 2017, 356 (6334), 194-197.

22. Seinen, A. B.; Driessen, A. J. M., Single-Molecule Studies on the Protein Translocon. Annu Rev Biophys 2019, 48, 185-207. 
23. Shine, J.; Dalgarno, L., The 3'-terminal sequence of Escherichia coli $16 \mathrm{~S}$ ribosomal RNA: complementarity to nonsense triplets and ribosome binding sites. Proc Natl Acad Sci U S A 1974, $71(4), 1342-6$.

24. Lee, K.; Holland-Staley, C. A.; Cunningham, P. R., Genetic analysis of the Shine-Dalgarno interaction: selection of alternative functional mRNA-rRNA combinations. RNA 1996, 2 (12), 1270-85.

25. Rackham, O.; Chin, J. W., A network of orthogonal ribosome x mRNA pairs. Nat Chem Biol 2005, 1 (3), 159-66.

26. Hui, A. S.; Eaton, D. H.; de Boer, H. A., Mutagenesis at the mRNA decoding site in the 16S ribosomal RNA using the specialized ribosome system in Escherichia coli. EMBO J 1988, 7 (13), 4383-8.

27. Jacob, W. F.; Santer, M.; Dahlberg, A. E., A single base change in the Shine-Dalgarno region of 16S rRNA of Escherichia coli affects translation of many proteins. Proc Natl Acad Sci U S A 1987, 84 (14), 4757-61.

28. Saito, K.; Green, R.; Buskirk, A. R., Translational initiation in E. coli occurs at the correct sites genome-wide in the absence of mRNA-rRNA base-pairing. Elife 2020, 9.

29. Okuyama, A.; Machiyama, N.; Kinoshita, T.; Tanaka, N., Inhibition by kasugamycin of initiation complex formation on 30 S ribosomes. Biochem Biophys Res Commun 1971, 43 (1), 196-9.

30. Schluenzen, F.; Takemoto, C.; Wilson, D. N.; Kaminishi, T.; Harms, J. M.; Hanawa-Suetsugu, K.; Szaflarski, W.; Kawazoe, M.; Shirouzu, M.; Nierhaus, K. H.; Yokoyama, S.; Fucini, P., The antibiotic kasugamycin mimics mRNA nucleotides to destabilize tRNA binding and inhibit canonical translation initiation. Nat Struct Mol Biol 2006, 13 (10), 871-8.

31. Jurenas, D.; Chatterjee, S.; Konijnenberg, A.; Sobott, F.; Droogmans, L.; Garcia-Pino, A.; Van Melderen, L., AtaT blocks translation initiation by N-acetylation of the initiator tRNA(fMet). Nat Chem Biol 2017, 13 (6), 640-646.

32. Forchhammer, J.; Lindahl, L., Growth rate of polypeptide chains as a function of the cell growth rate in a mutant of Escherichia coli 15. J Mol Biol 1971, 55 (3), 563-8.

33. Mangiarotti, G.; Apirion, D.; Schlessinger, D.; Silengo, L., Biosynthetic precursors of $30 S$ and $50 S$ ribosomal particles in Escherichia coli. Biochemistry 1968, 7 (1), 456-72.

34. Young, R.; Bremer, H., Polypeptide-chain-elongation rate in Escherichia coli $B / r$ as a function of growth rate. Biochem J 1976, 160 (2), 185-94.

35. Dai, X.; Zhu, M.; Warren, M.; Balakrishnan, R.; Patsalo, V.; Okano, H.; Williamson, J. R.; Fredrick, K.; Wang, Y. P.; Hwa, T., Reduction of translating ribosomes enables Escherichia coli to maintain elongation rates during slow growth. Nat Microbiol 2016, 2, 16231.

36. Schmidt, A.; Kochanowski, K.; Vedelaar, S.; Ahrne, E.; Volkmer, B.; Callipo, L.; Knoops, K.; Bauer, M.; Aebersold, R.; Heinemann, M., The quantitative and condition-dependent Escherichia coli proteome. Nat Biotechnol 2016, 34 (1), 104-10. 
37. Conway, T.; Creecy, J. P.; Maddox, S. M.; Grissom, J. E.; Conkle, T. L.; Shadid, T. M.; Teramoto, J.; San Miguel, P.; Shimada, T.; Ishihama, A.; Mori, H.; Wanner, B. L., Unprecedented highresolution view of bacterial operon architecture revealed by RNA sequencing. mBio 2014, 5 (4), e01442-14.

38. Nomura, M.; Gourse, R.; Baughman, G., Regulation of the synthesis of ribosomes and ribosomal components. Annu Rev Biochem 1984, 53, 75-117.

39. Oppenheim, D. S.; Yanofsky, C., Translational coupling during expression of the tryptophan operon of Escherichia coli. Genetics 1980, 95 (4), 785-95.

40. Rex, G.; Surin, B.; Besse, G.; Schneppe, B.; McCarthy, J. E., The mechanism of translational coupling in Escherichia coli. Higher order structure in the atpHA mRNA acts as a conformational switch regulating the access of de novo initiating ribosomes. J Biol Chem 1994, 269 (27), 1811827.

41. Huber, M.; Faure, G.; Laass, S.; Kolbe, E.; Seitz, K.; Wehrheim, C.; Wolf, Y. I.; Koonin, E. V.; Soppa, J., Translational coupling via termination-reinitiation in archaea and bacteria. Nat Commun 2019, 10 (1), 4006.

42. Adhin, M. R.; van Duin, J., Scanning model for translational reinitiation in eubacteria. J Mol Biol 1990, 213 (4), 811-8.

43. Yamamoto, H.; Wittek, D.; Gupta, R.; Qin, B.; Ueda, T.; Krause, R.; Yamamoto, K.; Albrecht, R.; Pech, M.; Nierhaus, K. H., 70S-scanning initiation is a novel and frequent initiation mode of ribosomal translation in bacteria. Proc Natl Acad Sci U S A 2016, 113 (9), E1180-9.

44. Jackson, R. J.; Hellen, C. U.; Pestova, T. V., The mechanism of eukaryotic translation initiation and principles of its regulation. Nat Rev Mol Cell Biol 2010, 11 (2), 113-27.

45. Gold, L.; Pribnow, D.; Schneider, T.; Shinedling, S.; Singer, B. S.; Stormo, G., Translational initiation in prokaryotes. Annu Rev Microbiol 1981, 35, 365-403.

46. Studer, S. M.; Joseph, S., Unfolding of mRNA secondary structure by the bacterial translation initiation complex. Mol Cell 2006, 22 (1), 105-15.

47. Milon, P.; Maracci, C.; Filonava, L.; Gualerzi, C. O.; Rodnina, M. V., Real-time assembly landscape of bacterial 30S translation initiation complex. Nat Struct Mol Biol 2012, 19 (6), 60915.

48. Li, G. W., How do bacteria tune translation efficiency? Curr Opin Microbiol 2015, 24, 66-71.

49. Hockenberry, A. J.; Stern, A. J.; Amaral, L. A. N.; Jewett, M. C., Diversity of Translation Initiation Mechanisms across Bacterial Species Is Driven by Environmental Conditions and Growth Demands. Mol Biol Evol 2018, 35 (3), 582-592.

50. Sigmund, C. D.; Ettayebi, M.; Morgan, E. A., Antibiotic resistance mutations in $16 \mathrm{~S}$ and $23 \mathrm{~S}$ ribosomal RNA genes of Escherichia coli. Nucleic Acids Res 1984, 12 (11), 4653-63.

51. Datsenko, K. A.; Wanner, B. L., One-step inactivation of chromosomal genes in Escherichia coli K-12 using PCR products. Proc Natl Acad Sci U S A 2000, 97 (12), 6640-5. 
52. Mutalik, V. K.; Guimaraes, J. C.; Cambray, G.; Lam, C.; Christoffersen, M. J.; Mai, Q. A.; Tran, A. B.; Paull, M.; Keasling, J. D.; Arkin, A. P.; Endy, D., Precise and reliable gene expression via standard transcription and translation initiation elements. Nat Methods 2013, 10 (4), 354-60.

53. Ranefall, P.; Sadanandan, S. K.; Wahlby, C., Fast Adaptive Local Thresholding Based on Ellipse fit. I S Biomed Imaging 2016, 205-208.

54. Loy, G.; Zelinsky, A., Fast radial symmetry for detecting points of interest. leee T Pattern Anal 2003, 25 (8), 959-973.

55. Jaqaman, K.; Loerke, D.; Mettlen, M.; Kuwata, H.; Grinstein, S.; Schmid, S. L.; Danuser, G., Robust single-particle tracking in live-cell time-lapse sequences. Nature Methods 2008, 5 (8), 695702. 


\section{SUPPLEMENTARY MATERIAL}

Supplementary Movie S1. Fluorescence microscopy data of L9-HaloTag labeled 50S ribosomal subunits, acquired with $3 \mathrm{~ms}$ laser exposures per $30 \mathrm{~ms}$ camera frame. Playback is 33 frames per second (i.e. realtime). The left panel shows raw data, whereas the right panel shows the cell outlines (segmented from phase-contrast images) as well as automatically detected diffusion trajectories, color-coded with respect to coarse-grained diffusion states estimated from HMM analysis. Diffusion trajectories were built in cells from the time-point where only one spot per cell remained in the current and all subsequent frames.
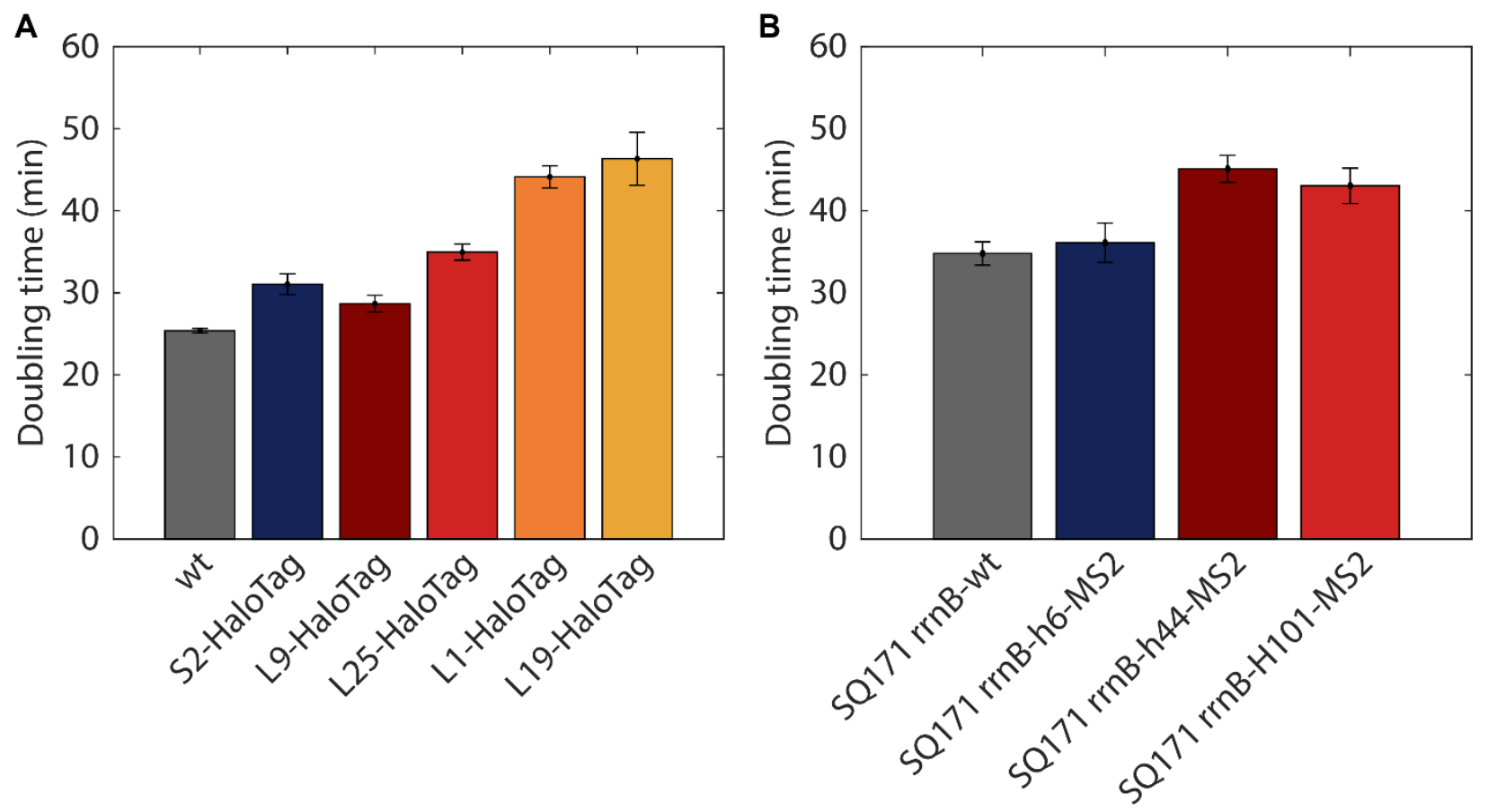

Fig. S1. Growth of $E$. coli strains constructed for tracking of ribosomal subunits. A. Doubling times of $E$. coli cells expressing S2-HaloTag, L1-HaloTag, L9-HaloTag, L19-HaloTag, L25-HaloTag, and isogenic E. coli MG1655. Error bars represent standard deviation calculated from 6 independent replicates. B. Doubling time of E. coli SQ171 strain which lacks all chromosomal rRNA operons and carrying plasmid pAMM552 expressing either the wt $r r n B$ operon or its mutated versions in which the MS2 aptamer was inserted in one of the helixes (helix 6, helix 44, or H101). Error bars represent standard deviation calculated from 6 independent replicates. 
bioRxiv preprint doi: https://doi.org/10.1101/2020.10.12.335505; this version posted October 12,2020 . The copyright holder for this preprint (which was not certified by peer review) is the author/funder, who has granted bioRxiv a license to display the preprint in perpetuity. It is made available under aCC-BY-NC-ND 4.0 International license.

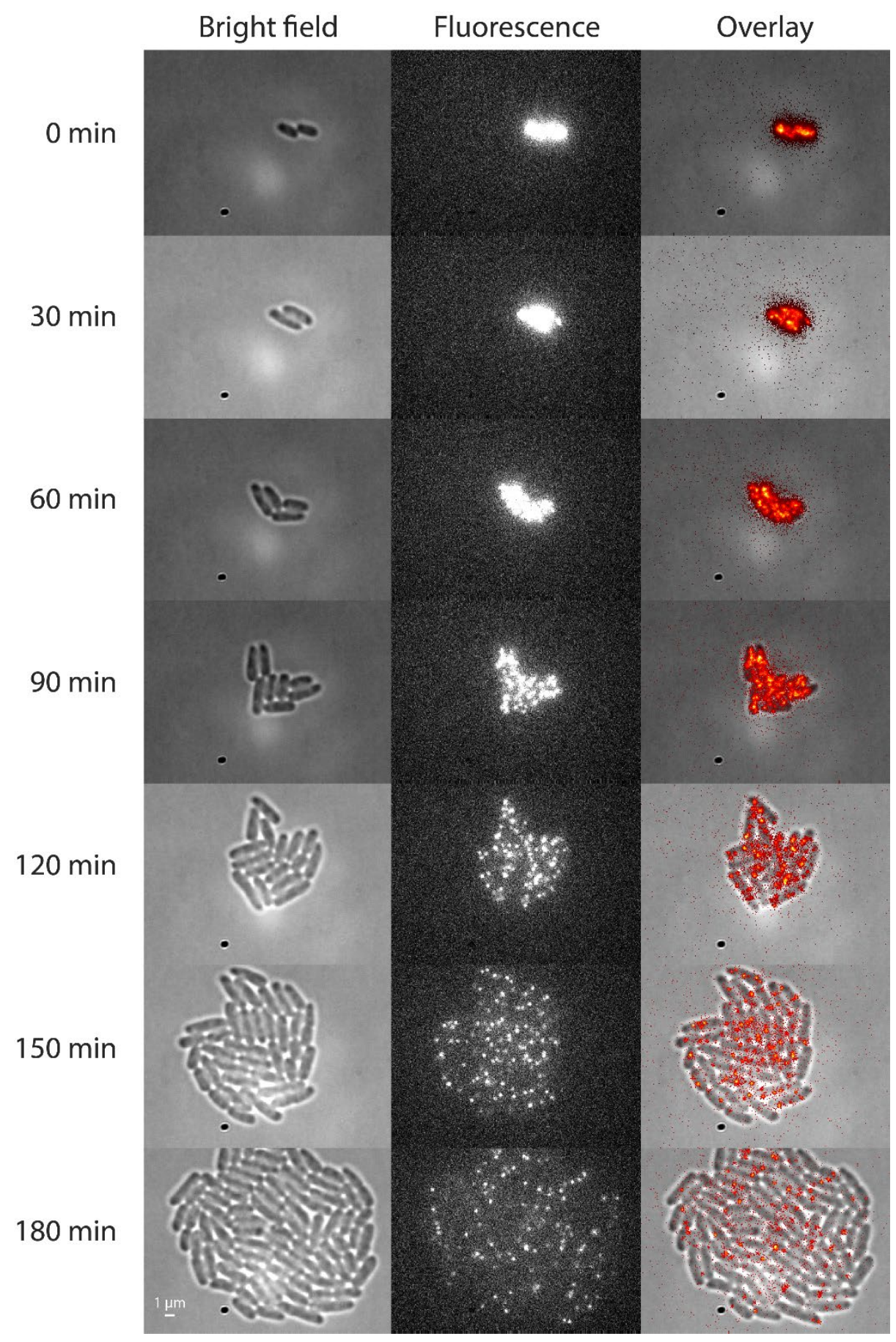

Fig. S2. Bright-field and fluorescence images (553 nm laser illumination) of L9-HaloTag expressing $E$. coli cells after labeling with the JF549 HaloTag ligand. 

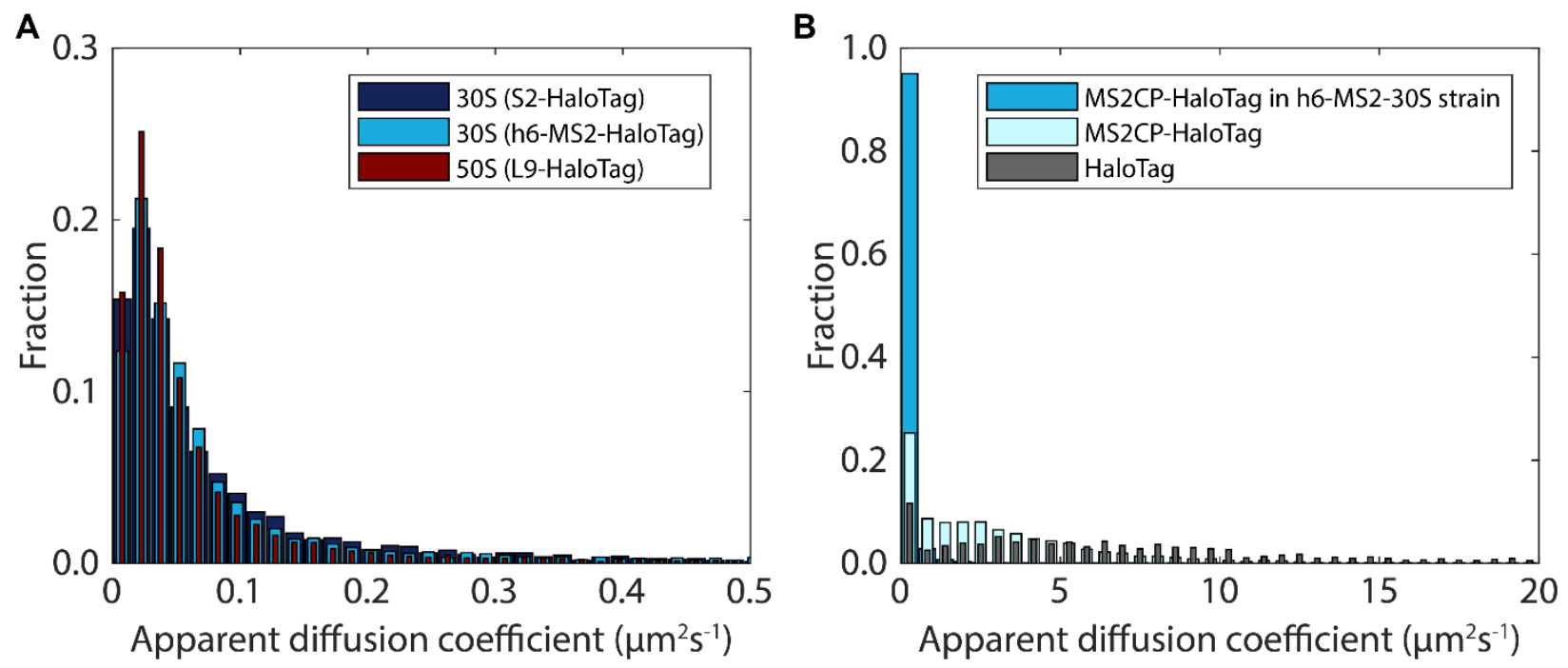

Fig. S3. Apparent diffusion coefficients estimated from mean-squared-displacement. A. Distribution of apparent diffusion coefficient for $30 \mathrm{~S}$ or $50 \mathrm{~S}$ HaloTag labeled ribosomal subunits. B. Distribution of apparent diffusion coefficient for free HaloTag and the MS2CP-HaloTag fusion in cells lacking the MS2 RNA aptamer. Data for diffusion of the MS2CP-HaloTag fusion in cells with the MS2 RNA aptamer inserted into a subpopulation of the 16S rRNA is shown for comparison. The average diffusion coefficient for HaloTag and the MS2CP-HaloTag were calculated to be $4 \mu \mathrm{m}^{2} / \mathrm{s}$ and $12 \mu \mathrm{m}^{2} / \mathrm{s}$, respectively. The apparent diffusion coefficients in both panels were estimated from mean-squared-displacement analysis of diffusion trajectory segments of 7 frames. Fluorescence data were acquired at $30 \mathrm{~ms}$ frames for panel $A$, and $5 \mathrm{~ms}$ for panel $\mathrm{B}$.

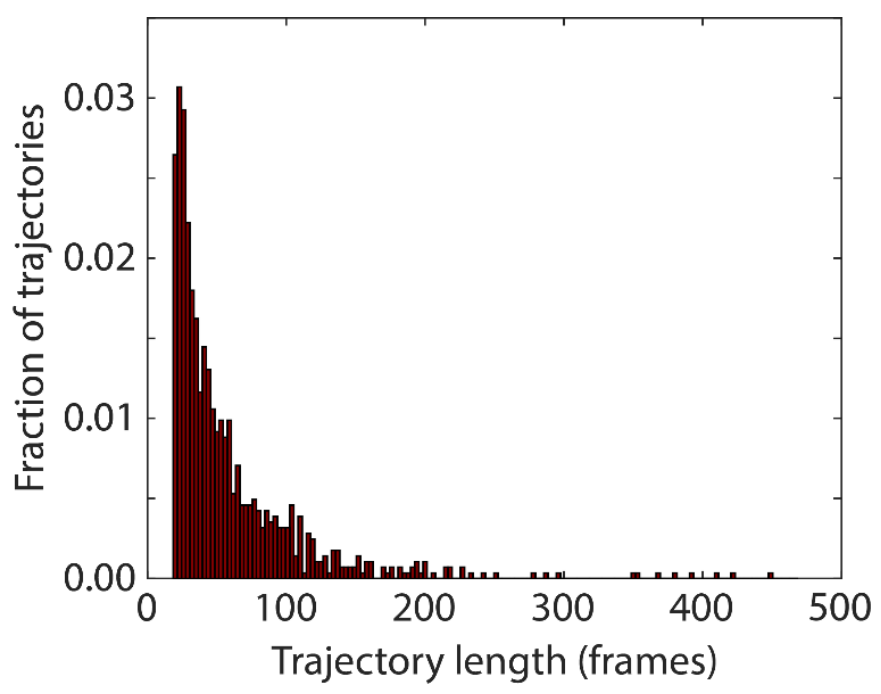

Fig. S4. Distribution of trajectory lengths from a single 50S (L9-HaloTag) tracking experiment. Only trajectories of length 20 frames or longer were saved for analysis. 


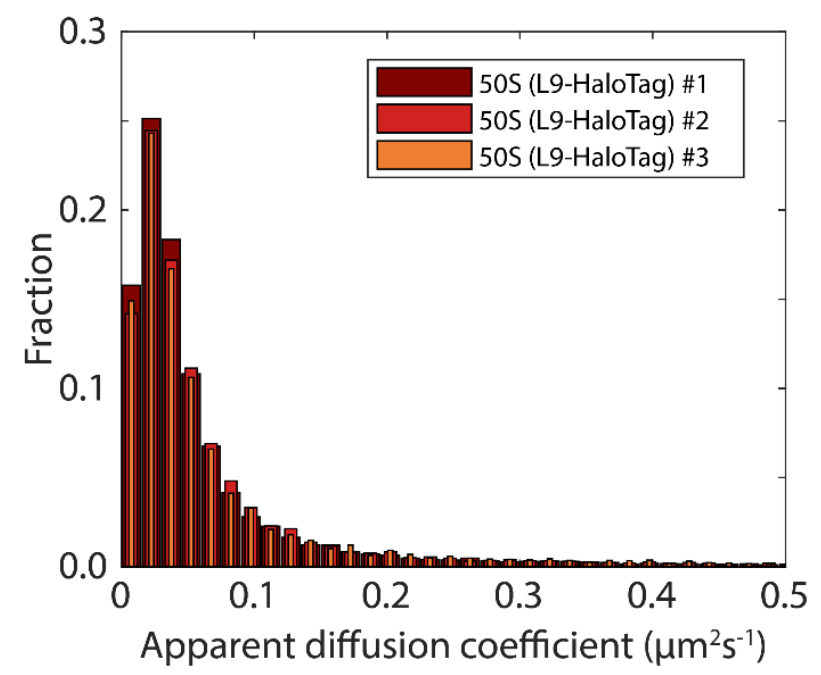

Fig. S5. Distribution of apparent diffusion coefficient for L9-HaloTag labeled 50S ribosomal subunits, estimated from mean-squared-displacement analysis of diffusion trajectory segments of 7 frames from three separate experiments.
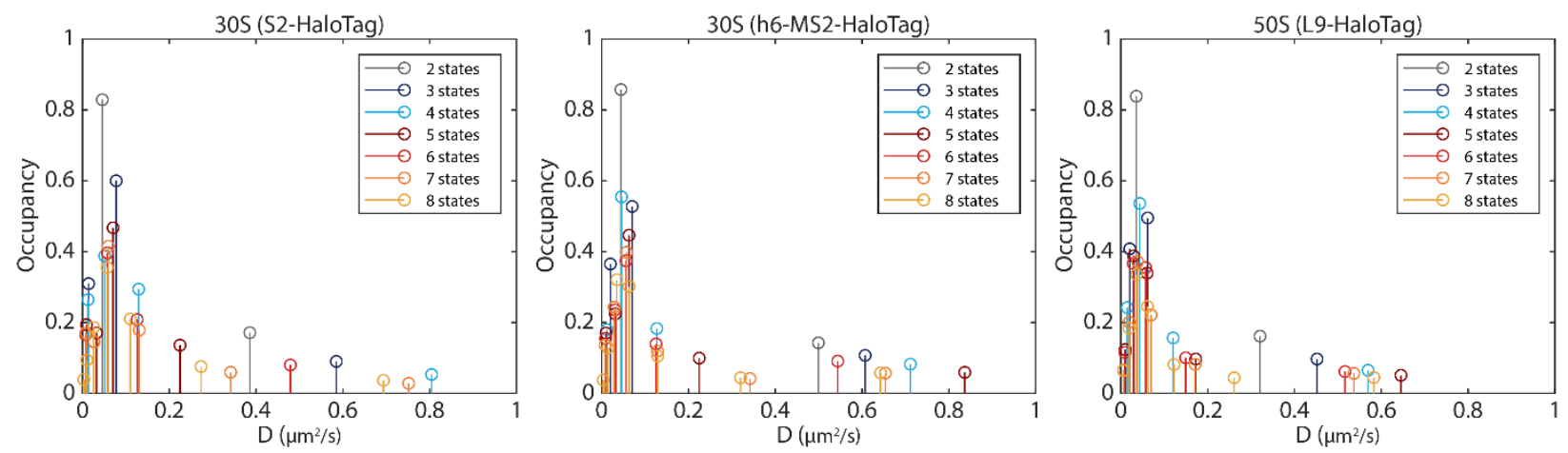

Fig. S6. HMM-estimated occupancies in different diffusion states for all fitted model sizes. All datasets, including low-occupancy states at $D>1 \mu \mathrm{m}^{2} / \mathrm{s}$, are also shown in Tables S4-S6. 